\title{
UNA MIRADA A LA SITUACIÓN CIENTÍFICA DE DOS ESPECIALIDADES ESENCIALES DE LA ENFERMERÍA CONTEMPORÁNEA: LA ANTROPOLOGÍA DE LOS CUIDADOS Y LA ENFERMERÍA TRANSCULTURAL
}

\begin{abstract}
Siles, J*; Cibanal, L*; Vizcaya, F***; Gabaldón, E**; Domínguez, J.M**; Solano, $\mathrm{C}^{* * *}$; García, $\mathrm{E}^{* * * *}$.
\end{abstract}

* Catedrático Dpto. Enfermería U.A. ** Titular Dpto. Enfermería U.A. *** Ayudante At. Primaria. Alicante. **** Enfermera At. Primaria. Alicante.

Dirección: Departamento de Enfermería, Universidad de Alicante.

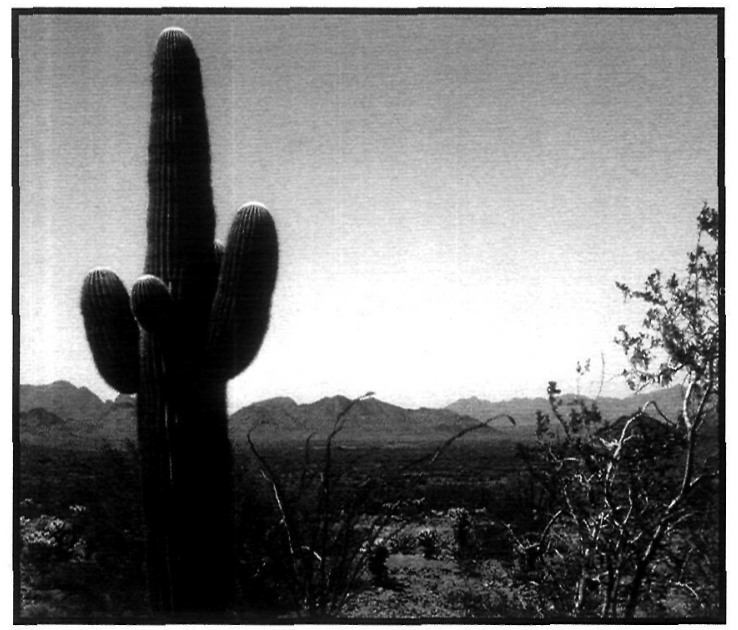

A LOOK INTO THE SCIENTIFIC SITUATION OF TWO ESSENTIAL SPECIALTIES OF CONTEMPORARY NURSING: ANTHROPOLOGY OF CARE AND TRANS-CULTURAL NURSING.

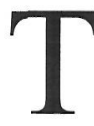
he general aim of this study is to explain the need for adopting transcultural methods and approaches within Iberian Nursing. To achieve this objective, it has been necessary to prove the anthropological nature of care and to identify the connection between the ideological frame of science and transcultural nursing.

Materials and method: The heuristic method was used to search and select convenient sources and materials for the study (nursing models with an anthropological and/or transcultural approach). These materials have been dealt with from the sociocritic and hermeneutic paradigm perspective, thus enabling us to explain the ideological factor within the scientific context.

Regarding results, the following models -which share the socioanthropological characteristics have been identified, described and explained: Madelaine Leininger's rising sun model; Larry Purnell's cultural competence model; Rachel Spector's cultural heritage and health traditions model.

Conclusions: for an adequate development of nursing science in a multicultural society, it is necessary to implement models that adopt anthropological approaches and consider the cultural and ideological factor as the object of study.

In current nursing there are models that comply with those characteristics (rising sun, cultural competence, heritage and health traditions).

\section{RESUMEN}

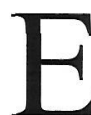
ste estudio tiene por objetivo general explicar la necesidad de adoptar enfoques y métodos transculturales en la enfermería ibérica. Para ello ha sido preciso demostrar la naturaleza antropológica de los cuidados e identificar la relación entre marco ideológico de la ciencia y 
la enfermería transcultural. Material y método: se ha empleado el método heurístico para la búsqueda y selección de fuentes y materiales pertinentes para el estudio (modelos de enfermería con enfoque antropológico y/o transcultural). Dichos materiales se han tratado desde la perspectiva del paradigma hermenéutico y sociocrítico posibilitando explicitar el factor ideológico en el contexto científico.

En cuanto a los resultados se han identificado, descrito y explicado desde la perspectiva de enfermería los siguientes modelos que comparten el carácter socioantropológico: el modelo de sol naciente de Madelaine Leininger; el modelo de competencia cultural de Larry Purnell; el modelo de herencia cultural y tradiciones de salud de Rachel Spector. Conclusiones: para el desarrollo adecuado de la disciplina enfermera en una sociedad multicultural es preciso implementar modelos que adopten enfoques antropológicos y consideren el factor cultural e ideológico como objeto de estudio. En la enfermería actual existen modelos que se ajustan a esas características (sol naciente, competencia cultural, herencia y tradiciones de salud).

\section{INTRODUCCIÓN}

Este estudio está orientado por un objetivo general que consiste en explicar la necesidad de adoptar enfoques y métodos transculturales en la enfermería ibérica. Para ello ha sido preciso alcanzar los siguientes objetivos específicos:

-Demostrar la naturaleza antropológica de los cuidados

-Explicar los modelos transculturales más reconocidos en enfermería.

-Identificar la relación entre marco ideológico de la ciencia y la enfermería transcultural

Se parte de las siguientes hipótesis iniciales:

-La enfermería es una disciplina cuya naturale$\mathrm{za}$ es fundamentalmente antropológica.

-La vertebración de la enfermería transcultural se demuestra mediante el desarrollo de los modelos, métodos y técnicas pertienentes para dicha subdisciplina.

-La antropología de los cuidados y la enfermería transcultural sólo pueden desarrollarse en un marco científico-ideológico democrático y sociocrítico.
Respecto al estado de la cuestión y las publicaciones específicas de antropología de los cuidados, sin duda alguna hay que empezar haciendo referencia obligada a Madeleine Leininger quien, en la década de los cincuenta, aplicó por primera vez el método antropológico a la enfermería. Fundó el campo "enfermería transcultural" mediante la fusión de dos conceptos que procedían de dos disciplinas distintas: la cultura (procedente de la antropología) y el cuidado (procedente de la enfermería) (LEININGER, 1978, 1995). Para que esta conjunción resultara armoniosa, la doctora Leininger ha realizado una serie de reflexiones sobre los conceptos esenciales de enfermería y antropología apoyando este trabajo teórico en un sólido y amplio abanico de trabajos de campo (LEININGER, 1970, 1988, 1991, 1995); y todo ello en el marco de los planteamientos éticos que toda actuación de enfermería implica (LEININGER, 1990). Rachel Spector ha profundizado en la dimensión hereditaria de la diversidad cultural (SPECTOR, 2000); Purnell desarrolló un modelo de enfermería basado en la competencia cultural (PURNELL, 1999), y otras autoras han aplicado el enfoque transcultural a trabajos de temática variada: la cultura homosexual (PACKIAO \& CARNEY, 2000).

Entre los seguidores más destacados de Leininger se encuentran: Rorbach, que ha trabajado la enfermería transcultural centrándose en la universalidad y la diversidad de los cuidados (ROHRBACH, 1996, 1998). Colliére ha contribuido de forma decisoria en el desarrollo de la antropología de los cuidados en Europa llamando la atención sobre el origen doméstico de la enfermería (COLLIÉRE, 1989, 1993).

En España Manuel Amezcua ha personificado la figura épica del pionero en estas lindes tan extrañas para la enfermería nacional hasta hace bien poco: además de demostrar la importancia de la antropología en la enfermería (AMEZCUA, 2000a), y aportar su experiencia en el proceso de adopción metodológica en el trabajo etnográfico (AMEZCUA, 2000b). El profesor Amezcua ha sido el fundador de la primera revista de enfermería -Index- que le ha prestado una atención prioritaria a la antropología de los cuidados. En esta misma línea, aunque cronológicamente mucho más 
reciente, hay que situar la aportación de Siles quien ha explicado epistemológicamente la naturaleza antropológica de la enfermería en su conjunto y del objeto-sujeto de la misma en particular (SILES, 1996, 1997), vinculando esta característica a la dimensión biológica-cultural de los cuidados (SILES, 1998), Asimismo, este mismo autor ha realizado aportaciones metodológicas como la equiparación del "campo" al texto, señalando la importancia de la antropología narrativa en los cuidados de enfermería (SILES, 2000). Otro de los enfermeros-antropólogos que ha contribuido a este proceso de vertebración disciplinar de la antropología de los cuidados es el profesor Valle (VALLE, 2000). Se recogen aportaciones más genéricas pero que apoyan la tendencia antropológica de los cuidados: desde la antropología hospitalaria y la cultura de la medicina y la actividad de curar (COMELLES, 1992; COMELLES y MARTÍNEZ et al, 1993). Sobe un tema tan candente como el mundo de las drogas resulta especialmente interesante el trabajo sobre antropología de la drogadicción (ROMANÍ, 1993). Otros trabajos se centran en: la antropología de la muerte (ALLUÉ, 1993) y antropología de la alimentación (CARRASCO, 1993).Paralelamente al incremento de trabajos de antropología de los cuidados han ido apareciendo las publicaciones que han servido de soporte y difusión de los mismos TABLA I):

En España, "Index de Enfermería" es fundada por el profesor Amezcua en 1988, constituyendo el órgano de expresión de la Fundación Index que incluso desarrolla actividades para potenciar la investigación enfermera como la base de datos "Cuiden".

Hay que esperar hasta 1997 para que vea la luz otra publicación dedicada a la antropología de los cuidados: "Cultura de los Cuidados", revista fundada por el profesor Siles y que constituye el instrumento de expresión de la Asociación de Historia y Antropología de los Cuidados. Hoy día, esta asociación y la fundación Index están hermanadas mediante un convenio en aras de alcanzar objetivos comunes.

A nivel internacional la primera revista específica de antropología de los cuidados es "Journal of Transcultural Nursing" que aparece en 1989 como órgano de difusión de la "Transcultural Nursing
Society" y bajo la influencia directa de la doctora Leininger. Actualmente, es dirigida por Marilyn Douglas. En la década de los noventa aparecen otras revistas relacionadas con enfoques antropológicos desde la perspectiva multicultural (Journal of Multicultural Nursing), y desde los presupuestos tan esenciales para la antropología y la educación como el enfoque holístico (Holistic Nursing Practice).

\section{DESARROLLO DEL TEMA}

1.- LA NATURALEZA ANTROPOLÓGICA DE LOS CUIDADOS

\subsection{LA PERSPECTIVA INTERPRETATIVA: UNA CUESTIÓN PREVIA}

La ciencia en general, pero la antropología en particular, captan y definen la realidad mediante interpretaciones. Ya Geertz equipara el antropólogo a un autor destinado a reescribir constantemente lo observado en el "campo" (GEERTZ, 1989). En consecuencia, la diversidad cultural es un hecho que hay que conjugar con una constante: la dualidad interpretativa de todos los fenómenos (SILES, 2000).

Los conceptos de "Emic" y "Etic" fueron acuñados por K.L. Pike para explicar el hecho de que cualquier fenómeno social, cultural, económico o sanitario tiene dos formas básicas de interpretación. Siguiendo al profesor Gustavo Bueno, se puede afirmar que cuando se tratan de reproducir los contenidos culturales tal como se les aparecen a los individuos que pertenecen a la cultura de referencia se está aplicando una perspectiva "emic". Por el contrario, cuando se trata de reproducir o describir los contenidos culturales teniendo en cuenta elementos, factores y conocimientos externos a los individuos que pertenecen a la cultura de referencia se está adoptando una perspectiva "etic". Cristobal Colón descubrió américa (perspetiva etic); Cristobal Colón llegó a las Indias navegando hacia Poniente (perspectiva emic).

\subsection{LA RELACIÓN ANTROPOLOGÍA-ENFER- MERÍA}

La vinculación entre disciplinas como antropología y enfermería, resulta tan natural que, paradójicamente puede pasar desapercibida, dado que el encuentro entre ambas se halla en su objeto de 
estudio: el ser humano desde una perspectiva holística, centrándose la antropología de los cuidados en el estudio integral del sistema de necesidades del hombre Un concepto esencial de antropología es aquel que la concibe como una ciencia del hombre que

- “(...) Se orienta a un conocimento global del hombre (...) aspirando a un conocimiento aplicable al conjunto del desarrollo humano (LEVISTRAUSS, 1993)

- "Ciencia que se encarga de estudiar al hombre inmerso en su cultura".

La naturaleza antropológica de los cuidados se comprende mejor analizando las relaciones entre la disciplina antropológica y la enfermera:

- Las dos disciplinas estudian la cultura como fuente de conocimientos desde una perspectiva holística.

Dicho esto, resulta ineluctable clarificar el concepto de cultura en el que están inmersas las situaciones vida-salud en cuyo plano adquieren sentido los cuidados de enfermería.

- La cultura puede ser definida de forma esencial como: "(...) El conjunto de los comportamientos, pensamientos y sentimientos implicados en el proceso de satisfacción de necesidades de un grupo humano (SILES, 2001)

Para delimitar la estrecha vinculación existente entre antropología y enfermería, una vez definidos los conceptos de antropología y cultura, resulta imprescindible definir el concepto más esencial de la disciplina enfermera: "el cuidado". Los cuidados no constituyen acciones ni pensamientos abstractos, sino que están inmersos en la cultura, no están monopolizados por enfermedades ni patologías, sino que forman parte del universo de los fenómenos cotidianos que se dan en cada cultura y se manifiestan en el conjunto de las situaciones "vida.salud", situaciones que se manifiestan en una relación triangular en la que los dos lados del triángulo están formados por la amplísima y variada dialéctica "salud-enfermedad", la cúspide o vértice superior está ocupado por la dimensión cultural y todo ello se asienta sobre la base de los cuidados (GRÁFICO I) . De forma esencial, el cuidado se puede definir como:

- El producto de la reflexión sobre las ideas, hechos y circunstancias (reflexión teórica, práctica y situacional) relacionadas con el proceso optimizador de las necesidades de salud que garantizan la integridad y la armonía de todas y cada una de las etapas que constituyen la vida humana" (SILES, 1996, 2000).

\section{EL VÍNCULO BIOLÓGICO DE PROYEC- CIÓN CULTURAL COMO CONFIGURADOR DE LOS CUIDADOS DE ENFERMERÍA}

La estrecha relación ente antropología y cuidados de enfermería se aprecia en la conexión existente entre los factores biológicos y la forma de organizar las actividades cotidianas que forman parte del proceso de satisfacción de necesidades. Desde tiempos ancestrales un factor biológico, el sexo, ha venido determinando la manera de organizar y distribuir las tareas entre los integrantes de los diferentes grupos humanos: la primigenia división sexual del trabajo (SILES, 1996, 1999). La mujer se encargaba de algo tan importante como la supervivencia del grupo mediante la reproducción. Desde los cuidados del embarazo al parto $y$, posteriormente, los cuidados perinatales, la lactancia y la crianza determinaron que la mujer fuera la responsable de los cuidados de enfermería. Es por todo ello que al hablar de cuidados de enfermería se hace referencia al género en particular y al mecanismo de satisfacción de necesidades en general. Esta situación de parentesco entre enfermería y antropología se refleja claramente al confrontar teorías tan disímiles, a priori, como las que a continuación se relacionan (GRÁFICO II):

-Las teorías de Carderera, un eugenista de principios del siglo XIX marca las pautas domésticas a las que debe ceñirse la labor de la mujer (GÓMEZ FERRER, 1987), y las clasifica en dos grupos "principios fundamentales" (orden, aseo y economía) y deberes domésticos (comida, vestido, limpieza).

- Las aportaciones esenciales de Florencia Nightingale que se recogen en su opúsculo "Notas de Enfermería..." publicado en 1859 (NIGHTINGALE, 1990) y describe la naturaleza de los cuidados: aire, luz, calor, limpieza, tranquilidad, dieta.

- La taxonomía del sistema de necesidades realizado por Henderson (HENDERSON \& BITE, 1988; HENDERSON, 1991). 
- La teoría de las necesidades básicas y sus concomitantes culturales de Malinoski (MALINOWSKI, 1984).

En todas estas clasificaciones y teorías en las que éstas se asientan, el sistema de necesidades y la forma de cumplir con este requisito elemental para todos los grupos humanos se aprecia una gran relación entre enfermería y antropología.

\subsection{LA DIFERENCIACIÓN ENTRE "ENFER- MERÍA TRANSCULTURAL Y ANTROPOLOGÍA DE LOS CUIDADOS"}

$\mathrm{Si}$, como se ha afirmado, la antropología se ocupa del estudio de la cultura, entendiendo a ésta como el conjunto de los comportamientos relacionados con el proceso de satisfacción de necesidades, la enfermería transcultural es, en realidad, una parte o especialidad antropológica, dado que enfatiza el estudio comparativo y sistemático intercultural para identificar las diferencias culturales que dan lugar a distintas formas de practicar y sentir los cuidados. Los conceptos de cultura y cuidado están estrechamente vinculados al origen de la enfermería transcultural, dado que, tal como señala Leininger, la E.T.C es:

"El área formal de estudio y trabajo centrado en el cuidado basado en la cultura" y con la finalidad de ayudar a las personas a: "mantener o recuperar su salud, hacer frente a sus discapacidades o a su muerte (LEININGER, 1978, 1995). Por ello Leiniger define el cuidado enfatizando su carácter diferenciador:

"...La esencia y acto diferenciador de la enfermería que permite a las personas mantener o restablecer su salud o bienestar o afrontar su enfermedad, su discapacidad o hacer frente a la muerte"(LEININGER, 1970, 1991, 1999)

\subsection{LOS MOMENTOS CULTURALES EN LAS SITUACIONES "VIDA-SALUD"}

Las transformaciones en las distintas situaciones de salud enfermedad provocan experiencias de las que se derivan distintas formas de interpretación del fenómeno. Básicamente se distinguen tres fases que son denominadas MOMENTOS CULTURALES y que responden a tramos o fases diferenciadas dentro del proceso situacional que se da en toda enfermedad.
A) Momento multicultural: responde a la fase en la que existe una muralla entre la forma de vida, las expectativas y la cultura anterior a la detección de la enfermedad (GRAFICO III). En esta fase resulta casi imposible la reconciliación con la forma de vida que exige la nueva situación: no se atienden las demandas producidas por el nuevo estado de satisfacción de necesidades, ni se pugna por implementar mecanismos compensatorios. Generalmente existe un sentimiento de rechazoodio por haber cambiado repudiando la nueva situación.

B) Momento intercultural: en esta fase se da un paso hacia delante, iniciándose el proceso de reconocimiento y conciliación con el nuevo estado de necesidades. En consecuencia empiezan a producirse brechas en la muralla (GRAFICOIV) y la persona se va adaptando a las nuevas exigencias que le impone la situación (comienza la lucha por establecer mecanismos compensatorios). Se podría decir que esta fase se corresponde con la de negociación.

C) Momento Transcultural: en este período se produce un ensanchamiento en la autoidentificación (toma de conciencia de la situación), reconociéndose en su nuevo estado. Ya no se odia a sí mismo y desaparece el sentimiento de rechazo (GRÁFICO V).

\section{2.-MODELOS EN ANTROPOLOGÍA DE LOS CUIDADOS Y ENFERMERÍA TRANSCULTU- RAL \\ 2.1. EL MODELO DEL SOL NACIENTE DE M.LEININGER.}

Este modelo es uno de los pioneros de la enfermería transcultural y representa una constelación que va de los cuidados culturales de la diversidad a los cuidados culturales a la universalidad (GRÁFICO VI). Está dividido en dos secciones: en la superior se tiene en cuenta desde la concepción del mundo, la estructura social y cultural, los valores culturales y modos de vida, contexto y medio ambiente, lenguaje y etnohistoria, influencias, expresiones de los cuidados, formas prácticas, familia y factores sociales, factores religiosos y filosóficos, factores tecnológicos, factores políti- 
cos y legales, factores económicos, factores racionales y el plano o base está la salud holística como bienestar.

En la sección inferior se parte de la consideración de grupos, familias, comunidades e instituciones, siguiendo tres círculos en los que se circunscriben a ambos lados los sistemas genéricos o populares, los sistemas profesionales y, en el centro, los cuidados de enfermería. Leininger toma de la enfermería los cuidados y de antropología la cultura. Estos dos conceptos se armonizan y complementan mediante el modelo del sol naciente, dado que, según el mismo, todos los cuidados tienen sus dimensiones culturales. Algunos de los presupuestos de este modelo transcultural son los siguientes (LEINIGER, 1999)

-Todos los seres humanos nacen, crecen y mueren y esperan ser cuidados según una perspectiva cultural.

- El cuidado culturalmente apropiado se convierte en significativo para las personas que están enfermas o sanas y es un potente generador de progresos curativos.

- Las enfermeras necesitan incorporar valores, creencias y modos de vida a sus cuidados para que mejoren y sean congruentes con los estilos de vida de las personas/grupos cuidados.

- El cuidado profesional culturalmente apropiado demuestra un gran potencial curativo. No puede haber curación sin cuidado.

- La recuperación de la enfermedad, la discapacidad o el afrontamiento de la muerte son situaciones que requieren que la enfermera conozca/comprenda la cultura de las personas a las que va a cuidar en cualquiera de los casos expresados.

- Aunque el cuidado integral y cultural resultan difíciles de captar/valorar, resultan críticos y decisivos para ayudar a las personas.

- Lo que más demandan las personas que van a un hospital o clínica es recibir unos cuidados adecuados por parte de las enfermeras, cuidados que demuestren conocimiento, comprensión y sensibilidad.

- Las enfermeras como principales protagonizadoras de los cuidados tienen un puesto preferencial para practicar la enfermería transcultural.

\subsection{EL MODELO DE COMPETENCIA CULTU- RAL DE PURNELL}

El modelo de Purnell se da a conocer en 1995 (PURNELL, 1999), se desarrolla mediante el empleo de razonamientos inductivo y deductivo y es, fundamentalmente, un instrumento para adaptar los cuidados al gran dinamismo de la sociedad actual. El proveedor de cuidados puede aplicar el modelo en sus niveles tres niveles: primario, secundario y terciario. Se basa en la forma de valorar las influencias culturales. Purnell denomina "características primarias y secundarias de diversidad" (TABLA II). El modelo es una consecuencia de la aplicación de teorías antropológicas, administrativas lingüísticas psicológicas, biológicas, religiosas y situaciones derivadas de las prácticas clínicas. Se representa mediante un diagrama (GRÁFICO VII) que es un círculo con un margen periférico que simboliza a la sociedad global, un segundo margen que representa a la comunidad y un tercer margen que representa a la familia. Por último un margen interno que representa a la persona. Este círculo, a su vez, se divide en 12 secciones o dominios culturales que tienen flechas bidireccionales dando a entender que no existen compartimentos estancos y que todos los dominios están relacionados y condicionados entre ellos mismos. El centro del modelo, que está vacío, representa aspectos desconocidos del grupo. En la parte inferior del modelo se aprecia una rueda dentada que representa el concepto de cultura-conciencia.

Los conceptos de metaparadigma que Purnell emplea en su modelo son los siguientes: la sociedad global, la comunidad, la familia, la persona y la salud. Estos conceptos son interpretados de forma genérica o esencial, sin considerar variantes raciales, culturales, nacionales, etc.). Asimismo, el círculo queda estructurado en doce dominios o sectores: herencia, comunicación, roles y organización familiar, asuntos laborales, ecología biocultural, comportamientos de alto riesgo, nutrición, embarazo y prácticas de nacimiento, rituales de muerte, espiritualidad, práctica cotidiana de cuidados de salud, proveedor del cuidado de salud. Por otro lado, para la fundamentación del modelo, el autor aporta 18 principios que van desde el reconocimiento de la necesidad de información relativa a la sociedad global, la comunidad, la familia, la 
persona y la salud al reconocimiento de que cada organización, asociación o profesión tienen su propia cultura. Se trata, en definitiva de un modelo flexible con un alto nivel de fluidez entre dominios y niveles.

\subsection{EL MODELO DE HERENCIA CULTURAL Y TRADICIONES DE SALUD DE RACHEL SPEC- TOR}

Este modelo parte del presupuesto de la importancia que tienen las tradiciones en los comportamientos relacionados con los cuidados de salud. Para Spector, es imprescindible tomar en consideración el contexto y los límites culturales en los que vive el individuo, la familia o la comunidd (SPECTOR, 1999). Asimismo, Spector opina que el dispensador de cuidados debe reunir los siguientes requisitos para llegar a comprender realmente lo que hace y que, a la vez, su práctica profesional resulte eficaz (TABLA III).

-Ser culturalmente sensible, lo cual implica adquirir los conocimientos necesarios para comprender las tradiciones relacionadas con la salud y la enfermedad. Pero no basta con los conocimientos, dado que para alcanzar cierta sensibilidad es preciso desarrollar actitudes favorables a dicho sentimiento.

-Ser culturalmente congruente; es decir, tener la capacidad a llevar a la práctica los conocimientos que se poseen de forma adecuada y rentabilizarlos desde el punto de vista de la enfermería mediante la dispensación de cuidados coherentes culturalmente y de gran calidad profesional.

-Ser culturalmente competente implica prestar atención de forma adecuada al paciente teniendo en cuenta el contexto y manteniendo, por tanto, la filosofía holística de los cuidados.

Spector utiliza el proceso de enfermería como base para su modelo, pero a las 5 fases del P.E. les añade otros tres (valoración del legado cultural, fenómenos culturales que afectan a la salud y, por último, valoración de las tradiciones de salud).

-El legado cultural, su nivel de consistencia, es valorado mediante el nivel de importancia de la cultura, la etnicidad y la religión en la vida del individuo, la familia o la comunidad.

-Los factores que afectan a la salud son seis (Spector sigue los estudios de Giger y
Davidhizard, 1995) Orientación en el tiempo, espacio personal y territorialidad, comunicación, organización social, variables biológicas, hábitos de comida y alimentación, control del entorno

-Las tradiciones de salud constituyen un modelo enfocado holísticamente (atención

físicamental y espiritual), integrado por 8

dimensiones interrelacionadas:

-Creencias y prácticas para el mantenimiento de salud.

-Creencias y prácticas para la protección de la salud.

-Actividades relacionadas con la restauración de la salud.

-Salud y creencias tradicionales

-Salud y prácticas tradicionales.

-Remedios tradicionales.

-Salud y sanadores tradicionales.

-Cuidados culturales y proceso de enfermería.

3.- EL MARCO IDEOLÓGICO DE LA CIENCIA Y LA ENFERMERÍA TRANSCULTURAL

3.1 ENFERMERIA EN CONTEXTO DEL PARADIGMA RACIONAL TECNOLÓGICO

Heredero del positivismo, se caracteriza por consagrar a la objetividad el proceso investigador, constituyendo el enfermero un mero aplicador de técnicas y el paciente un receptor de las mismas. En este tipo de paradigmas se identifica lo real con lo científico constituyendo el baluarte principal del mismo la objetividad, hunde sus raíces en la cuantificación, la verificación-falsación-replicabilidad (PALAZÓN, 1991). Existe un notable ensalzamiento de la actividad nomotética (búsqueda de normas y leyes). En el terreno de la enfermería, la perspectiva del paradigma sociocrítico ha sido abordada por diversos autores (SILES Y GARCÍA, 1995a; SILES, 1997c) (TABLA IV).

\subsection{ENFERMERÍA EN CONTEXTO DEL PARADIGMA INTERPRETATIVO}

Desde este paradigma se concibe el proceso de investigación como un intervalo subjetivo (tanto por parte del sujeto investigador como del objetosujeto investigado). El conocimiento teórico es fruto de la interacción que tiene efecto en la práctica de la disciplina dada (cuidado, educación, procedimiento). Desde los planteamientos de este 
paradigma el enfermero-a se considera un agente comunicador e interpretador de la realidad del paciente. Las características del paradigma interpretativo en el contexto enfermero han sido estudiadas por diversos autores (SILES Y GARCÍA, 1995; SILES, 1997) (TABLA V).

\subsection{ENFERMERÍA EN CONTEXTO DEL PARA- DIGMA SOCIOCRÍTICO}

El paradigma crítico se caracteriza por el carácter dialéctico-crítico del proceso del conocimiento y por la toma en consideración del papel preponderante de la ideología en el proceso científico. La función y el fin fundamental de los presupuestos de esta plataforma los constituyen la emancipación participativa, y las actividades y las tareas en los procesos acontecen en la práctica y son socialmente significativas. La acción práctica es crítica y colaborativa. Habermas es el investigador que más ha contribuido al desarrollo del paradigma crítico. El enfermero-a se considera como un agente de cambio sociosanitario (SILES Y GARCÍA, 1995; SILES, 1997) (TABLA VI).

\subsection{A MODO DE RESUMEN ACERCA DEL DEBATE IDEOLOGIA-PARADIGMAS CIENTÍFICOS}

Los tres paradigmas aquí analizados ofrecen, desde sus respectivos presupuestos, un marco ideológico en el cual la actividad investigadora en enfermería puede tener cabida, aunque de forma muy diversa.

-En el marco del paradigma racional tecnológico no tienen cabida ni enfoque holístico ni el componente humanístico tan inherentes para la disciplina enfermera, la antropología y la enfermería transcultural. El paradigma racional puede ser útil en parcelas muy importantes para el desarrollo de los cuidados enfermeros (las dimensiones biológicas, fisiológicas, etc.). La ideología neopositivista ha sido convertida en una especie de liturgia y el aparato ortopédico con el que se pretende interpretar la realidad deja fuera de la ciencia la parte más importante de la disciplina enfermera. El paradigma racional tecnológico responde a la época técnica de la enfermería cuya ideología es también técnica y, por ende, de carácter subalterno: enfermero ejecutor (ATS) que depende de otros profesionales para actuar y que concibe el proceso de salud enfermedad como una realidad natural y externa (PALAZON, 1991: 199). En el contexto no tiene cabida el enfoque holístico imprescindible para la disciplina enfermera, la antropología y la enfermería trans-cultural. En enfermería, la asunción de este paradigma produce la tylorización de los enfermeros que se constituyen en meros ejecutores de programas diseñados en las direcciones de los grandes centros burocráticos de la Administración Sanitaria.

Esta tylorización produce los siguientes efectos:

-Descualificación de los profesionales mediante el control técnico (tiempos de ejecución de tareas estandarizadas) que repercute en una progresiva automatización-deshumanización de la actividad desarrollada.

-Entorpecimiento de cualquier intento de innovación en un ontextopredeterminado desde un puesto de control externo (metáfora: hombre como hardware biológico y software psicológico (PALAZON, 1991: 199-210).

- El paradigma interpretativo simbólico implica la adopción de una ideología que permite a los profesionales de enfermería actuar como facilitadores-cooperadores con el sujeto (paciente) respecto la optimización de su proceso salud-enfermedad. La naturaleza del conocimiento respecto del proceso salud-enfermedad no es objetiva, sino subjetiva y la acción de enfermería se desarrolla en un clima de interacción y constante intersubjetividad teniendo en cuenta la realidad específica del paciente. En este marco, es posible el desarrollo de enfoques holísticos tan perentorios para la disciplina enfermera, la antropología y la enfermería transcultural.

La exigencia de un estatuto de objetividad impide la comprensión de significaciones sustanciales para el proceso salud-enfermedad. Manteniendo la distancia respecto del sujeto sobre el que se actúa no se logra una verdadera imagen del objeto de la investigación.

-El paradigma crítico se desarrolla en un marco ideológico-científico que concibe la imagen de un profesional de la salud más comprometido con el cambio (como organizador de actividades críticas e innovadoras respecto del proceso salud-enfermedad). La naturaleza del conocimiento de enferme- 
ría es dialéctico-crítica. El paralelismo entre marginación política y laboral se mantuvo durante mucho tiempo en el terreno de la enfermería. La concienciación por parte de las enfermeras de su doble condición discriminatoria, en la que participaron activamente asociaciones y organismos tales como el CIE (Consejo Internacional de Enfermería), constituyó el primer paso en la larga lucha por cambiar esa situación práctica. Sin duda alguna, es en el contexto del paradigma crítico en el que de forma más pertinente y eficaz se pueden desarrollar enfoques, métodos y técnicas consustanciales para la disciplina enfermera, la antropología y la enfermería transcultural.

En nuestra opinión, el paradigma que evidencia más la necesidad de la licenciatura y que mejor se ajusta a las características de la Enfermería, en razón de su Objeto-Sujeto (el ser humano en el contexto de los cuidados convergentes en el intervalo salud-enfermedad), y del enfoque holístico que se propugna baluarte de todo acto de enfermería, es el sociocrítico o interpretativo simbólico. Desde los presupuestos de esta plataforma se potencia la interactividad precisa para que el enfermero-a lleve adelante la comprensión de cada situación específica e inherente a cada individuo o grupo de ellos. De cualquier manera no se trata de adoptar un paradigma como si fuera un acto de fe. La complejidad de la práctica en enfermería hace recomendable una integración crítica, razonada y pertinente de distintos paradigmas de acuerdo con la naturaleza de los problemas planteados y sus respectivas situaciones (SILES, 1997).

\section{RESULTADOS Y SU DISCUSIÓN}

Aunque existe todavía cierta ambigüedad respecto al papel que le corresponde desarrollar a la enfermería en el marco de la antropología de los cuidados y la enfermería transcultural -ambigüedad fomentada desde el interior de la enfermería, pero sobre todo desde otras disciplinas-, parece evidente que poco a poco, debido al trabajo de esforzados grupos de enfermeros de diferentes países que se han unido en para clarificar el papel de la enfermería transcultural se va saliendo de esa situación tan poco positiva y paralizante.

A la luz de la evolución experimentada por la enfermería transcultural en las dos últimas déca- das, se puede afirmar que se ha producido la consolidación de dicha especialidad de la enfermería antropológica. Este proceso de vertebración de la enfermería transcultural se evidencia en:

-Los encuentros celebrados con la finalidad de intercambiar conocimientos e ideas: organización de eventos científico-sociales, tales como los que anualmente patrocina la Transcultural Nursing Society en países de todo el mundo (en el año 2004 se celebrará en España), los encuentros anuales organizados por la Fundación Index y los bianuales celebrados por la Asociación de Historia y Antropología de los Cuidados.

-Los instrumentos de difusión de las investigaciones desarrolladas en el marco de la antropología de los cuidados y la enfermería transcultural, es decir las revistas especializadas en la temática antropológica y transcultural: Transcultural Nursing, Multicultural Nursing, Index de Enfermería y Cultura de los Cuidados.

-El factor de cohesión sociocorporativa en el contexto de la enfermería transcultural se evidencia en el estrechamiento de las relaciones que se ha producido entre las diferentes asociaciones comprometidas con la temática transcultural: Transcultural Nursing Society, Fundación Index y Asociación de Historia y Antropología de los Cuidados.

\section{CONCLUSIONES}

-La enfermería es una disciplina cuya naturaleza es fundamentalmente antropológica.

-En las dos últimas décadas se ha producido la vertebración de la enfermería transcultural mediante el desarrollo de los modelos, métodos y técnicas pertinentes para dicha subdisciplina.

-Los modelos de enfermería transcultural más difundidos son los aportados por autores que viven en países multiétnicos y multiculturales: Leiniger (modelo del sol naciente); Spector (modelo de herencia cultural y tradiciones de salud); Purnell (modelo de competencia cultural).

-La antropología de los cuidados y la enfermería transcultural sólo pueden desarrollarse en un marco científico-ideológico democrático y sociocrítico.

-El enfoque holístico y los métodos y técnicas relativos a dicho enfoque, sólo pueden aplicarse en el marco de una ideología científica en el que tenga cabida el paradigma sociocrítico. 


\section{BIBLIOGRAFÍA}

ALLUÉ, M. (1993) La antropología de la muerte. Rol de enfermería, 179-180: 33-40.

AMEZCUA, M. (2000a) Antropología de los cuidados. Enfermedad y padecimiento. Significados del enfermar en la práctica de los cuidados. Cultura de los Cuidados, IV/7-8: 60-67

AMEZCUA, M. (2000b) El trabajo etnográfico en salud. Una aproximación a la observación participante. Index de Enfermería, XI/30: 30-35.

CARRASCO, S. (1993) Comportamiento alimentario. Rol de enfermería, 179-180: 13-18.

COLLIÉRE, M.F. (1989) Utilización de la antropología para abordar las situaciones de cuidados.Rol, 179/180: 17-25

COLLIÈRE, M.F. (1993) Promover la vida: de la práctica de las mujeres cuidadoras a los cuidados de enfermería. MacGraw-Hill/Interamericana, Madrid.

COMELLES, J.Mª (1992) Cuidar y curar. Rol de enfermería, 172: 35-40.

COMELLES, J. $M^{a}$ y MARTínez, A, (et. Al) (1993) Enfermedad, cultura y sociedad : un ensayo sobre las relaciones entre la antropología social y la medicina Ediciones de la Universidad Complutense, Madrid

GEERTZ, C. (1989) El antropólogo como autor. Paidós, Barcelona.

GIGER, J. \& DAVIDHIZARD, R. (1995) Transcultural nursing intervention. Mosby, San Louis

GOMEZ FERRER, G. (1987) El trabajo doméstico en los manuales escolares (contribución al conocimiento de las mentalidades de las clases medias). Actas de Jornadas de Investigación Interdisciplinar. Universidad Autónoma, Madrid:136-147

HENDERSON, V. \& NITE, G. (1988) Enfermería: teoría y práctica. Interamerica-na, México.

HENDERSON, V. (1991) The nature of nursing: reflections after 25 years. National League for Nursing, New York.

LEINIGER, M. (1970) Nursing and anthropology. Two worlds to blend. John Wiley \& Sons, New York.

LEINIGER, M. (1978) Transcultural nursing; concepts, theories and practices. John Wiley \& Sons, New York.

LEININGER, M. (1990) Ethical and moral dimensions of care. Wayne State University Press, Detroit,

LEININGER, M. (1991) Culture care. Diversity and universatily. A theory of nursing. John Wiley \& Sons, New York,.

LEINIGER, M. (1988) Care: the essence of nursing and health care. Wayne State, University Press, Detroit.
LEININGER, M. (1995) Transcultural nursing: concepts, theories and practices. McGraw-Hill, Columbus.

LEVI-STRAUSS, C. (1993) Estructura social. En: BOHANANN, P. \& GLAZER, M. Antropología. Lecturas, 440-473. MacGrawHill, Madrid.

LEININGER, M. (1999) Cuidar a los que son de culturas diferentes requiere el conocimiento y las aptitudes de la enfermería transcultural. Cultura de los Cuidados, 5: 5-8.

MALINOWSKI, B. (1984) Una teoría científica de la cultura. Sarpe, Madrid. Manual Moderno, México.

NIGHTINGALE, F. (1990) Notas sobre Enfermería. ¿Qué es y qué no es? Salvat, Barcelona.

PALAZÓN, F. (1991) El educador ¿tecnólogo o investigador? Anales de Pedagogía, 9: 197-241.

PURNELL, L. (1999) El modelo de competencia cultural de Purnell: descripción y uso en la práctica, educativa, administración e investigación. Cultura de los Cuidados, III/6: 91-102.

ROMANÍ, O. (1993) Antropología y drogodependencias. Rol de enfermería, 179-180:19-26.

ROHRBACH, C. (1996) Reflexión sur le diversité dans les soins. Revue soins infirmiers, 7: 69-73.

ROHRBACH, C. (1998) Introducción a la teoría de los cuidados culturales enfermeros, de la diversidad y de la universalidad de Madelaine Leininger. Cultura de los Cuidados, 3: 41-45.

SILES, J. et al (1992a) La Enfermería Comparada: un instrumento para canalizar y sistematizar las experiencias y conocimientos de una profesión transnacional. Enfermería Científica, 124/ 125: 16-19.

SILES, J. (1992b) La formación de los profesionales de Enfermería en España: pasado, presente y futuro. Publicación científica de Enfermería, 1: 4-7.

SILES, J. (1994) Evolución histórica del corporativismo de Enfermería. La influencia de los colegios en el proceso de socialización profesional. Index III/10: 11-15.

SILES, J. y GARCÍA, E. (1995) Las características de los paradigmas científicos y su adecuación a la investigación en enfermería. Enfermería Científica 160/161: 10-15.

SILES, J. (1996a) Pasado, presente y futuro de la enfermería en España: Perspectiva histórica y epistemológica. CECOVA/ Fundación José Llopis, Alicante.

SILES, J. (1996b) Pasado, presente y futuro de la enfermería en España. Perspectiva histórica y epistemológica. CECOVA/Fundación José Llopis, Alicante. 
SILES, J. (1997) Epistemología y enfermería: por una fundamentación científica y profesional de la disciplina. Enfermería Clínica. Enfermería Clínica: 4/7: 188-194.

SILES, J. et al (1998) El eslabón biológico en la historia de los cuidados de salud. El caso de las nodrizas: una visión antropológica de la enfermería. Index de Enfermería, II/20-21: 16-.

SILES, J. et al (1999a) Enfermería y fenomenología: niveles de especificidad en el conjunto de la producción científica biomédica. "Enfermería Clínica" Enero-Febrero.

SILES, J. (1999b) Historia de la enfermería comunitaria en España. Un enfoque social, político, científico e ideológico de la evolución de los cuidados comunitarios. Index, VIII/24-25: 25-30.
SILES, J. (1999c) Historia de la Enfermería. Aguaclara, Alicante.

SILES, J. (2000) Antropología narrativa de los cuidados. CECOVA, Alicante.

SPECTOR, R. (2000a) Cultural diversity in health and illness. Prentice Health, Upper SAdle Brook.

SPECTOR, R. (2000b) Cultural care: Guides to heritage assessment. Prentice Health, Uppers Sadle Brook.

SPECTOR, R. (2000c) La enfermería transcultural: pasado, presente y futuro. Cultura de los Cuidados, IV/7-8: 116-127

VALLE, I. (2000) Cuidar desde una perspectiva cultural. Cultura de los Cuidados, IV:7-8: 96-101.

\section{MODELO SUNRISE}

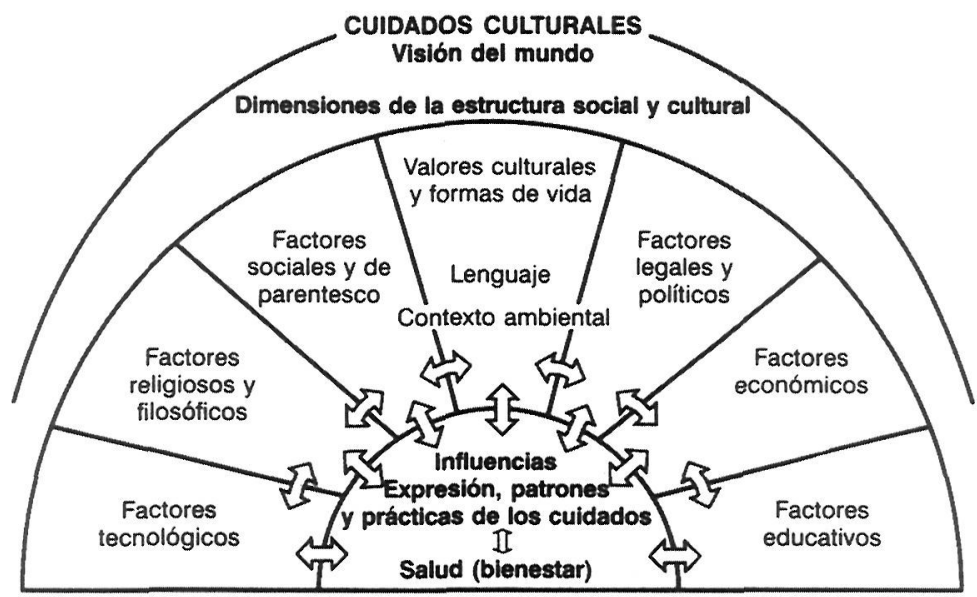

Individuos, familias, grupos, comunidades e instituciones en distintos sistemas de salud

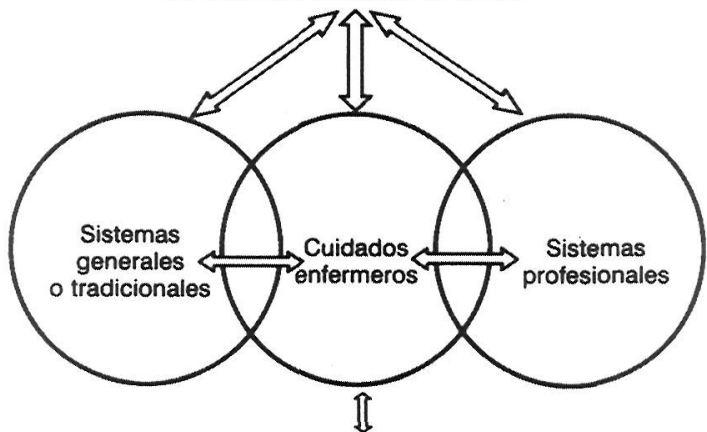

Decisiones y actuaciones de los cuidados enfermeros<smiles>[CH]</smiles>

Preservación y mantenimiento de los cuidados culturales Acomodación y negociación de los cuidados culturales Remodelación y reestructuración de los cuidados culturales 


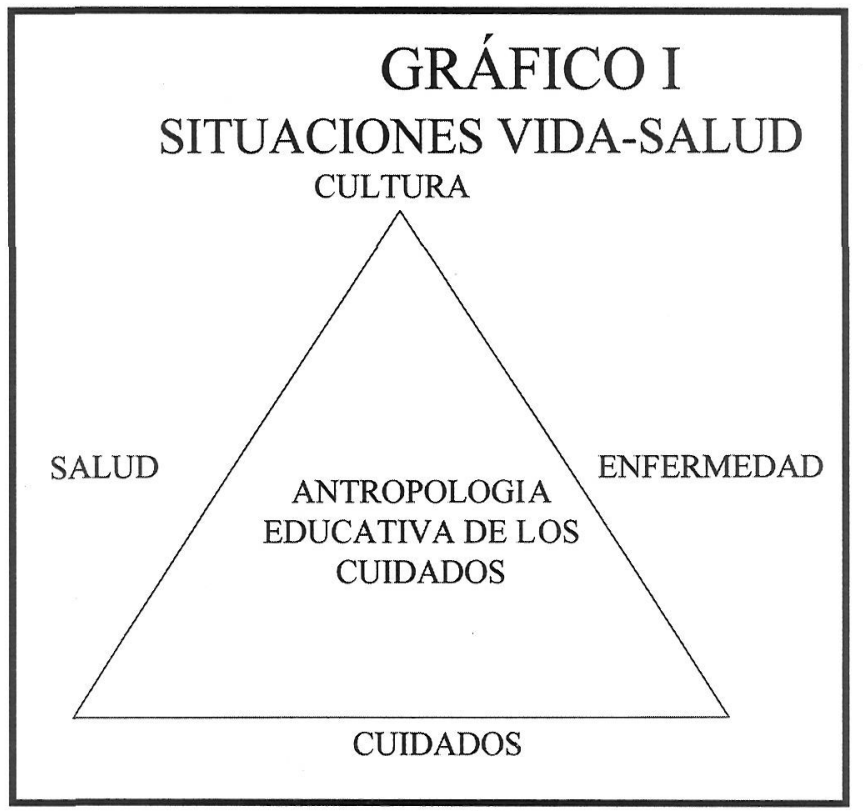

GRÁFICO II

ESLABÓN BIOLÓGICO-CULTURAL DE LOS CUIDADOS

ROLES BIOLOGICOS

CON PROYECCIÓN CULTURAL
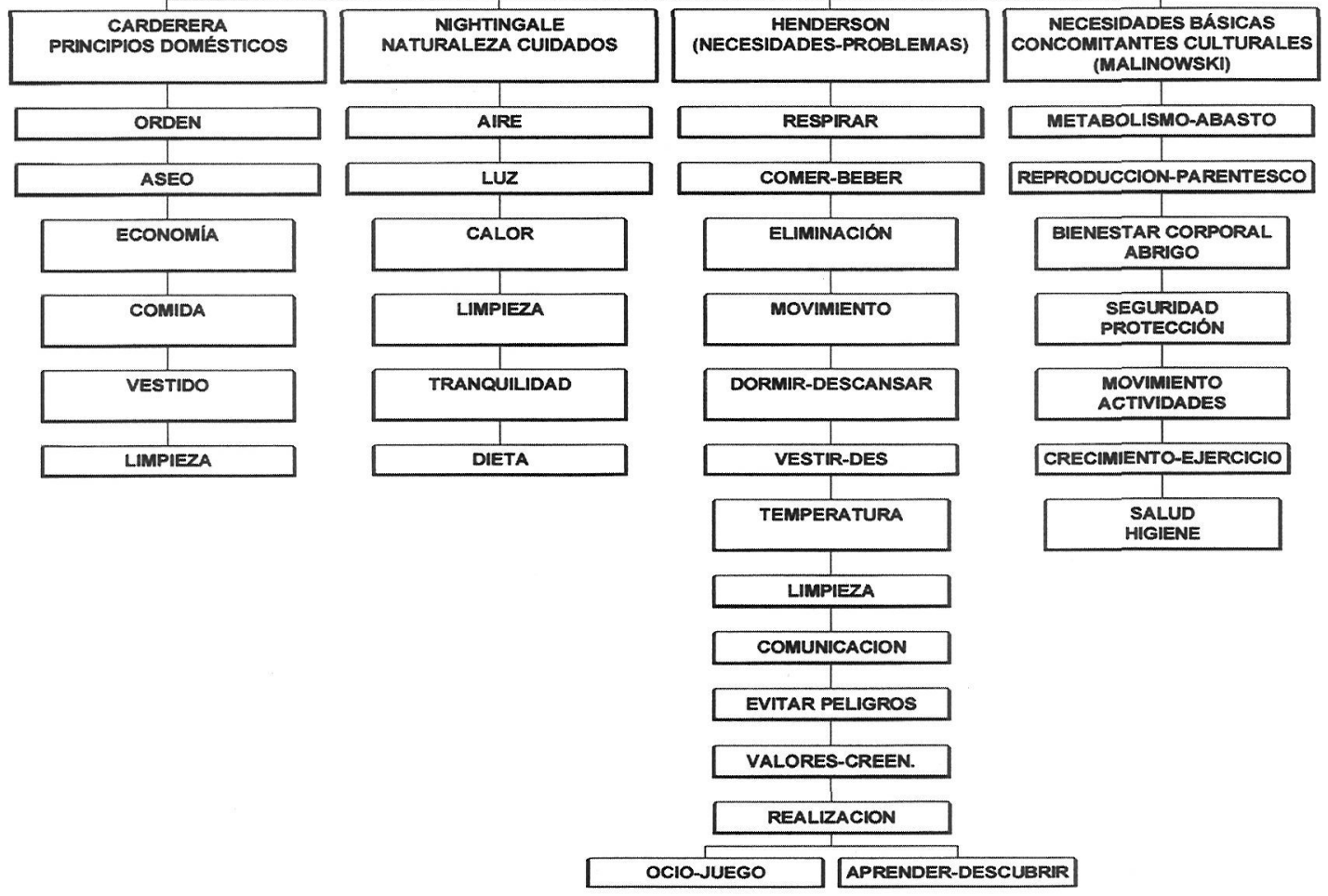

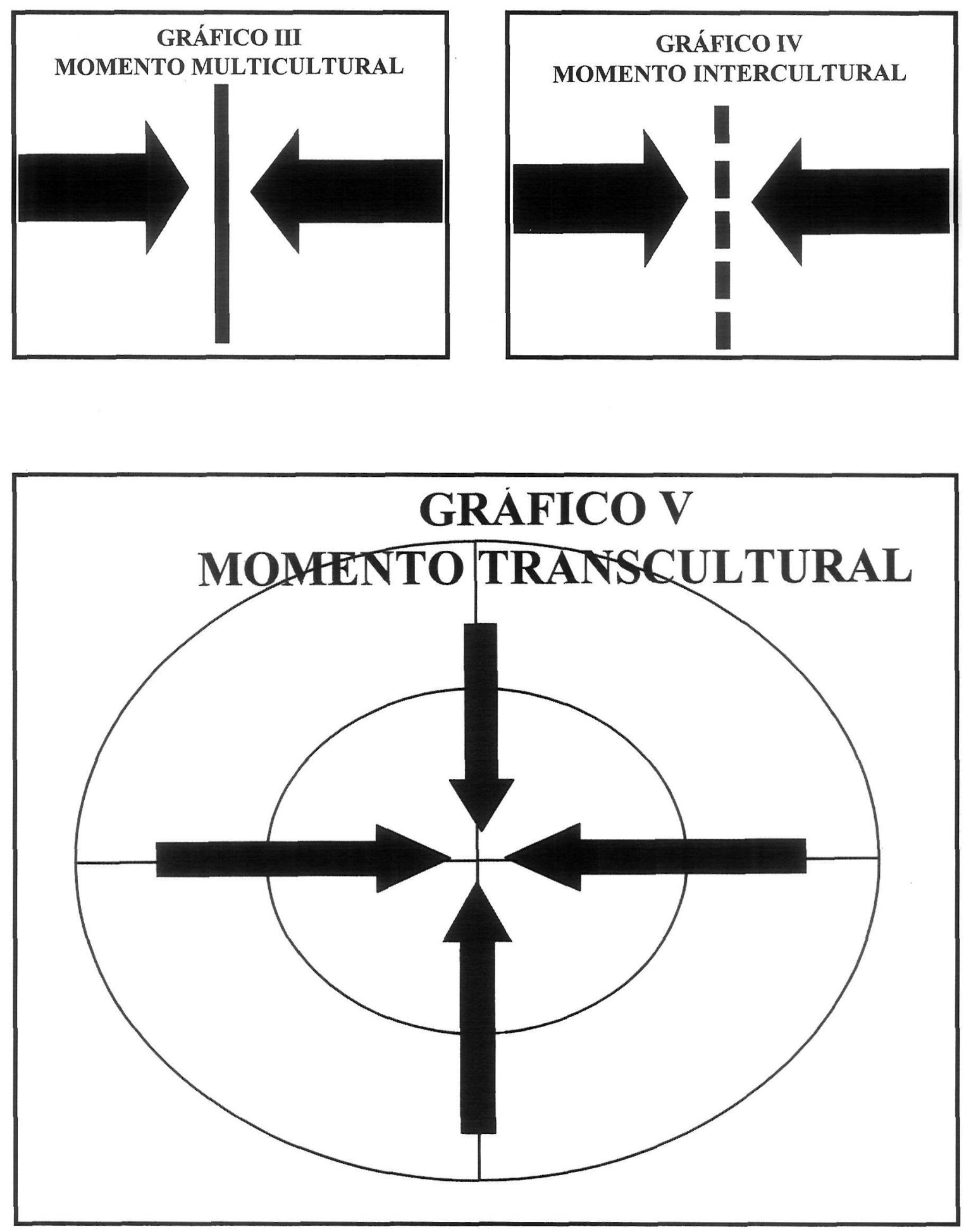


\section{TABLA I \\ PUBLICACIONES DE ANTROPOLOGÍA DE LOS CUIDADOS}

ESPAÑOLAS

-INDEX

DE ENFERMERÍA.

(1988) (http//www.doc6.es/index

-CULTURA DE LOS CUIDADOS

(1997) (http:// http://culturacuidados. ua.es)

INTERNACIONALES

-JOURNAL OF TRANSCULTURAL NURSING (1989)

(http://www.culturenu rse.org/main/mainindex.html)

-JOURNAL OF MULTICULTURAL NURSING

-HOLISTIC NURSING PRACTICE

Fuente: Bases de datos (Cuiden, Medline: IM, INI); Directorio revistas

biomédicas españolas, Centro de Documentación e informática biomédica,

Universidad de Valencia.

TABLA II

CARACTERISTICAS $1^{\text {a }}$ Y $2^{\mathrm{a}}$ DE DIVERSIDAD

CARACTERÍSTICAS PRIMARIAS

-NACIONALIDAD

RAZA

-COLOR PIEL

GÉNERO

$-E D A D$

RELIGIÓN

\section{CARACTERÍSTICAS SECUNDARIAS}

-E/.SOCIOECONÓMICO

-EXP.MILITAR

-CREENCIAS POLÍTICAS

-ESTADO PATERNALISTA

-ORIENTACIÓN SEXUAL

-TIEMPO FUERA DEL PAÍS
NIVEL EDUCACIÓN

OCUPACIÓN

RESIDENCIA URBANA/RURAL

CARACTERES FÍSICOS

ESTADO CIVIL

ASUNTOS DE GÉNERO

-RAZONES Y SITUACIÓN DE LA MIGRACIÓN (LEGAL, INDOCUMENTADO, ETC.) 


\section{TABLA III \\ MODELO DE HERENCIA CULTURAL Y TRADICIONES DE SALUD (SPECTOR)}

-Ser culturalmente sensible (tener los conocimientos necesarios para comprender las tradiciones relacionadas con la salud y la enfermedad)

-Ser culturalmente congruente (tener la capacidad a llevar a la práctica los conocimientos que se poseen de forma adecuada)

-Ser culturalmente competente implica prestar atención de forma adecuada al paciente teniendo en cuenta el contexto y manteniendo, por tanto, la filosofía holística de los cuidados.

\section{TABLA IV \\ E.T.C EN EL PARADIGMA TECNOLÓGICO}

-AGENTE CONTROLADOR DEL PROCESO.

ENFERMERO-A

-MANIPULADOR EXTERNO Y OBJETIVO RESPECTO DEL ESTADO DE SALUD PACIENTE Y SUS NECESIDADES.

-APLICADOR DE PRESCRIPCIONES.

-ADMINISTRADOR TERAPÉUTICO Y TÉCNICO.

PACIENTE

-OBJETO DE CONTROL Y DESTINATARIO DE PRESCRIPCIONES TERAPÉUTICAS -ACATAMIENTO PASIVO DE NORMAS, PRESCRIPCIONES TERAPÉUTICAS Y PROCEDIMIENTOS DE ENFERMERIA

CONCEPTO DE SALUD

-CONCEPTO NEGATIVO/VACÍO: SALUd COMO AUSENCIA DE ALGO, OMISIÓN DE MANIFESTACIONES CLÍNICAS OPRIVACIÓN DE SINTOMATOLOGÍA.

TIPO DE CONOCIMIENTO CONSTRUIDO

-CIENCIA NATURAL HEREDERA DEL NEOPOSITIVISMO

-CARÁCTER OBJETIVO DE LA ACTIVIDAD CIENTIFIFICA

-CONTROL EXTERNO DEL. PROCESO.

¿CÓMO SE CONSTRUYE?

-INVESTIGACión EXPERIMENTAL (PRUEBAS ESTANDARIZADAS, TEST, ENCUESTAS). -GRAN PROTAGONISMO DE LA INSTRUMENTACIÓN ESTADISTICA. -PROCESO CIENTÍFICO AJENO Y SUPERIOR AL OBJETO-SUJETO (PACIENTE INVESTIGADO CUIDADO).

-LA TEORÍA DIRIGE LA ACCIÓN SEPARADA DE LA PRAXIS.

- -LA ENFERMERÍA SE ENFOCA COMO UN PROCESO TECNOLÓGICO.

-ENFERMERO-A COMO EJECUTOR DE TÉCNICAS. 


\section{TABLA V \\ E.T.C. EN EL PARADIGMA HERMENEUTICO}

ENFERMERO

-AGENTE DE COMUNICACIÓN COMPRENSIVA E INTERACTIVA. -INTERPRETADOR DE LA REALIDAD DEL PACIENTE

PACIENTE

-SUJETO COMUNICADOR DE SU ESTADO Y SUS EXPECTATIVAS (INTERPRETACIÓN PERSONAL DE SU SITUACIÓN).

\section{CONCEPTO DE SALUD}

-CONCEPTO SITUACIONAL ELABORADO INTERACTIVAMENTE (PACIENTE ENFERMERO)

TIPO DE CONOCIMIENTO CONSTRUIDO

-ENFERMERIA COMO CONSTRUCCIÓN PERSONAL

-SOCIOLOGÍA INTERACCIONISTA.

-HERMENÉUTICA Y FENOMENOLOGÍA DE LOS CUIDADOS.

-ANTROPOLOGÍACULTURAL.

¿CÓMO SE CONSTRUYE?

-INVESTIGACIÓN ETNOGRÁFICA.

-ESTUDIO DE SIGNIFICADOS EN SUS CONTEXTOS (USO DE MÉTODOS Y TÉCNICAS FENOMENOLÓGICAS, NARRATIVAS, MATERIAL BIOGRÁFICO Y AUTOBIOGRÁFICO)

-OBSERVACIÓN PARTICIPANTE, NOTAS DE CAMPO, DIARIOS, RELATOS, ETC.

$$
\text { ¿PARA QUÉ SIRVE? }
$$

-LA PRÁCTICA ES EL FUNDAMENTO DE LA TEORÍA (SU PRESUPUESTO) -ENFERMERIA ENFOCADA COMO PROCESO DE INTERCOMUNICACIÓN ENFERMERO PACIENTE.

-INTERVENCIÓN PRÁCTICA INTERPRETATIVA. ACTO DE COMPRENSIÓN

TABLA VI

E.T.C EN EL PARADIGMA SOCIOCRITICO

-AGENTE DE CAMBIO SOCIOSANITARIO

ENFERMERO-A

PACIENTE

-SUJETO IMPLICADO EN SU PROPIO PROCESO DE CAMBIO DE ESTADO DE SALUD Y SATISFACCIÓN DE NECESIDADES.

-CONCEPTO POSITIVO.

CONCEPTO DE SALUD

-REELABORACIÓN DELMISMO POR PARTE DEL SUJETO.

TIPO DE CONOCIMIENTO CONSTRUIDO

-ENFERMERÍA COMO CONSTRUCCIÓN SOCIAL Y SANITARIA.

-DIALÉCTICA-SUBJETIVIDAD

CONSTRUCCIÓN NUEVAS REALIDADES.

-INVESTIGACIÓN CRÍTICA

¿CÓMO SE CONSTRUYE?

-ESTUDIOS DE CONTEXTOS PERSONALES Y SOCIAES.

-COMPROMISO PARA SOLUCIÓN DE PROBLEMAS.

-LOS SUJETOS (PACIENTES) POR ENCIMA DE LOS MÉTODOS.

$$
\text { ¿PARA QUÉ SIRVE? }
$$

-ENFERMERIA ENFOCADA COMO INTERVENCIÓN EN PRÁCTICA SOCIAL Y ANTROPOLÓGICA DEL SUJETO.

-EL PROCESO DE TOMA DE DECISIONES CENTRADO EN LA CAPACIDAD CRIITICA. 